\title{
Donafenib-Loaded Callispheres Beads Embolization in a VX2 Liver Tumor: Investigating Efficacy, Safety, and Improvement of Tumor Angiogenesis After Embolization
}

\author{
Tongqiang $\mathrm{Li}^{1,2, *}$ \\ Qin Shi ${ }^{1,2, *}$ \\ Jiacheng Liu (D) ${ }^{1,2, *}$ \\ Yingliang Wang ${ }^{1,2}$ \\ Chen Zhou',2 \\ Chaoyang Wang ${ }^{1,2}$ \\ Shuguang Ju ${ }^{1,2}$ \\ Songjiang Huang ${ }^{1,2}$ \\ Chongtu Yang ${ }^{1,2}$ \\ Yang Chen ${ }^{1,2}$ \\ Yaowei Bai iD ${ }^{1,2}$ \\ Bin Xiong $\mathbb{D}^{1,2}$

\begin{abstract}
'Department of Radiology, Union Hospital, Tongji Medical College, Huazhong University of Science and Technology, Wuhan, 430022, People's Republic of China; ${ }^{2}$ Hubei Province Key Laboratory of Molecular Imaging, Wuhan, 430022, People's Republic of China

*These authors contributed equally to this work
\end{abstract}

Correspondence: Bin Xiong Department of Radiology, Union Hospital, Tongji Medical College, Huazhong University of Science and Technology, Jiefang Avenue \#I277, Wuhan, 430022, People's Republic of China

Email herr_xiong@I26.com
Objective: To investigate the efficiency and safety of callispheres beads loaded with donafenib (DCBs) for embolization in a VX2 liver tumor, as well as the improvement of tumor angiogenesis following embolization.

Methods: Forty New Zealand white rabbit VX2 liver tumors were treated with four different drugs via the hepatic artery: NS (normal saline), CB (blank callispheres beads), ACB (adriamycin-loaded callispheres beads) and DCB (DCBs). Hematoxylin-eosin staining was performed to assess tumor necrosis, while MRI was employed to detect the changes in tumor size. The safety was evaluated by the liver and kidney function parameters, and the immunofluorescence and immunohistochemical staining were performed to reflect the tumor hypoxia and tumor angiogenesis following embolization.

Results: The DCB group had the smallest tumor growth rate, but the tumor necrosis rate was the highest of the four groups. Compared to the $\mathrm{CB}$ and ACB groups, the DCB group did not aggravate the liver damage and had no influence on kidney function. The staining results showed that, although the tumor hypoxia deteriorated after DCBs embolization, the expression of VEGF (vascular endothelial growth factor) reduced, thus inhibiting tumor angiogenesis.

Conclusion: DCB administration via hepatic artery is an effective and safe treatment for a preclinical liver cancer model, with the unique benefit of suppressing tumor angiogenesis following embolization.

Keywords: hepatocellular carcinoma, donafenib, transcatheter arterial chemoembolization, tumor angiogenesis, efficacy, safety

\section{Introduction}

Transcatheter arterial chemoembolization (TACE) is currently the most commonly used treatment for unresectable hepatocellular carcinoma (HCC). ${ }^{1,2}$ However, the residual tumor after TACE can enhance tumor angiogenesis by upregulating hypoxia-inducible factor-1 $\alpha$ (HIF-1 $\alpha$ ) and vascular endothelial growth factor (VEGF) expression, ${ }^{3,4}$ leading to tumor progression or recurrence. ${ }^{5-7}$ In recent years, several studies have revealed that the high expression of VEGF after TACE could be antagonized by VEGF antibodies or antiangiogenic drugs, ${ }^{8-10}$ but how and when to combine antiangiogenic drugs with TACE is still an open question. ${ }^{11}$ Interestingly, in the clinical trials which have been published, one of the key distinctions between the successful TACTICS trial and the prior fail trials 
was the time to apply sorafenib. ${ }^{12,13}$ In fact, there is already evidence that the most suitable regimen for administering sorafenib is before VEGF expression increases. ${ }^{14}$

In addition to directly suppressing the growth of tumor cells through downregulation of the Ras/Raf/Mek/ Erk signaling pathway, sorafenib also exerts an anticancer effect by suppressing angiogenesis via inhibiting VEGF receptor (VEGFR-1, 2, 3) and platelet-derived growth factor receptor (PDGFR) ${ }^{15}$ Donafenib, a novel oral small-molecule multikinase inhibitor, ${ }^{16}$ was developed by substituting a trideuteriomethyl group for a methyl on a sorafenib molecule, ${ }^{17}$ potentially enhancing molecular stability for an improved pharmacokinetic profile. ${ }^{18}$ Donafenib was considered to work in a similar way to sorafenib in terms of anti-cancer activity. ${ }^{17}$ An open-label, randomized, parallel-controlled, multicenter Phase II-III trial (ZGDH3) conducted in China showed that, when compared to sorafenib, donafenib had a significantly longer median overall survival (12.1 vs 10.3 months, $\mathrm{P}=0.0245$ ) and appeared to have improved tolerability. ${ }^{16}$ Based on this research, donafenib has been approved for first-line treatment of advanced HCC in China in June 2021.
In this study, we established donafenib-loaded callispheres beads (DCBs), in which donafenib could be sustained released in a VX2 liver tumor model. After embolization, we looked into the efficiency, safety and improvement of tumor angiogenesis.

\section{Materials and Methods Materials}

Donafenib tosylate prodrug (Zelgen Medicine Co. Ltd., Jiangsu, China) was dissolved in a mixture of $25.0 \%$ polyethylene glycol (PEG400, Sinopharm Chemical Reagent Beijing, China), 25.0\% ethyl alcohol (Sinopharm Chemical Reagent Beijing, China) and 50.0\% distilled water to obtain $5 \mathrm{mg} / \mathrm{mL}$ stock solution. Callispheres beads (CB) samples with 100-300 $\mu \mathrm{m}$ diameter range (Hengrui Medicine Co. Ltd., Jiangsu, China) were used for the study.

\section{DCBs Characterizations}

Donafenib has a similar chemical formula to sorafenib, ${ }^{18}$ and both carry a positive charge $\left(\mathrm{NH}^{+}\right)$(Figure 1A). Composed of polyvinyl alcohol hybrid hydrogel linked to sulfonate group, $\mathrm{CBs}$ are net-like spheres carrying negative<smiles>CNC(=O)c1cc(Oc2ccc(NC(=O)Nc3ccc(Cl)c(C(F)(F)F)c3)cc2)ccn1</smiles>
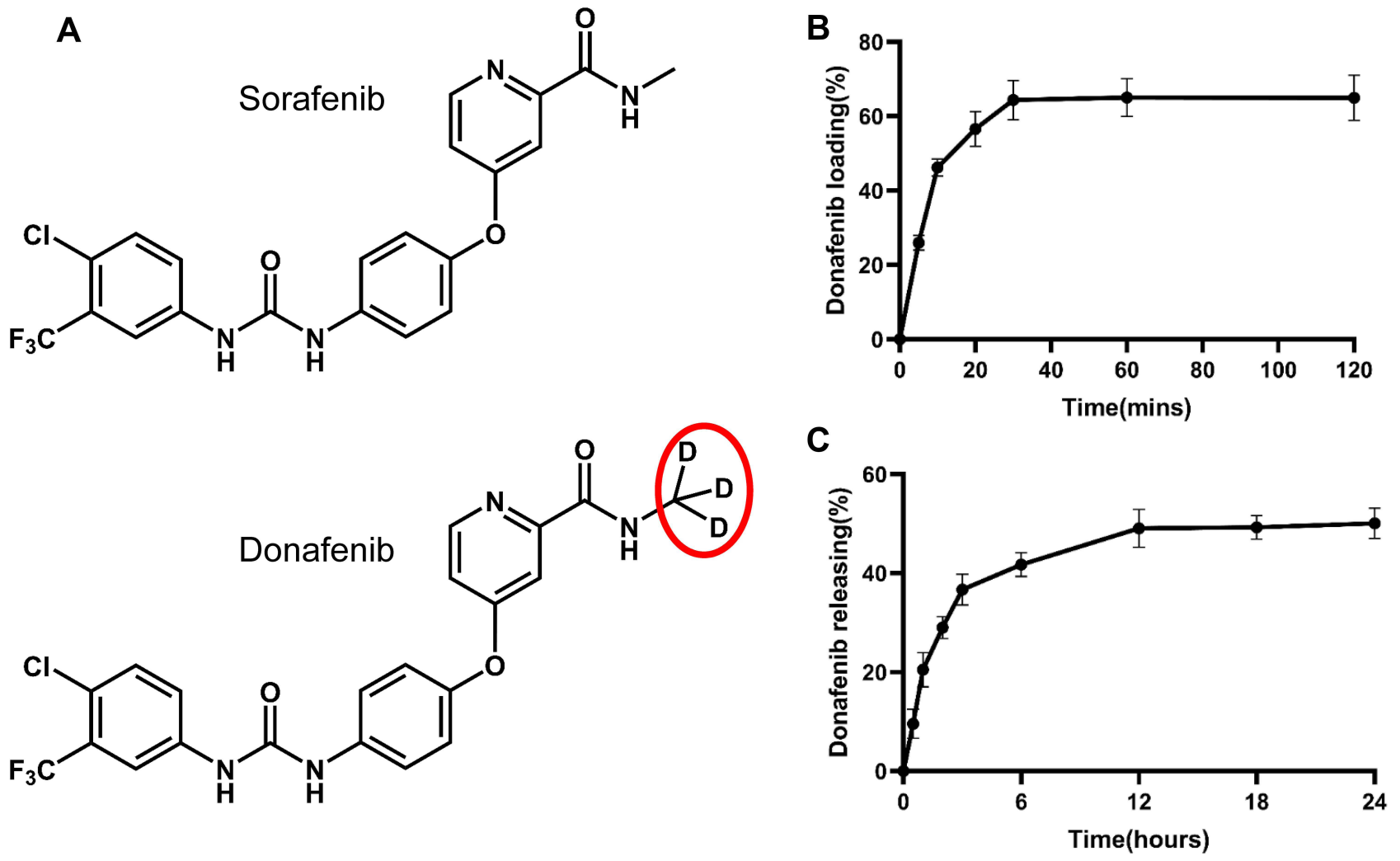

Figure I (A) Chemical formula of sorafenib and donafenib; (B) loading profiles of 100-300 $\mu \mathrm{m}$ CBs with a maximal loading of $20 \mathrm{mg}$ donafenib/g beads; (C) percentage curve of donafenib release. 
charge, ${ }^{19,20}$ allowing drugs charged positively for interaction. The DCBs characterizations were explored as described in the previous literature. ${ }^{20,21}$ Specifically, the donafenib concentration was detected by a multimode plate reader (BioTek Epoch, USA) at $305 \mathrm{~nm}$. The loading and releasing efficiency were indirectly calculated by the donafenib concentration of the supernatants, which were obtained from the mixture system at different times. All experiments were performed in triplicate.

\section{The Loading Efficiency}

100-300 $\mu \mathrm{m}$ CBs ( $1 \mathrm{~g}$ ) and the stock solution containing $20 \mathrm{mg}$ of donafenib were kept for 120 minutes in a shaker at room temperature. At $5 \mathrm{~min}, 10 \mathrm{~min}, 20 \mathrm{~min}, 30 \mathrm{~min}, 60$ min and $120 \mathrm{~min}$, an equal amount of supernatant was collected from mixtures.

\section{The Releasing Efficiency}

The DCBs were suspended in $10 \mathrm{~mL}$ of normal saline containing 1\% Tween 80 (Solarbio Technology Co. Ltd., Beijing, China) and incubated at $37^{\circ} \mathrm{C}$ on a programmable incubator shaker (SPH-1038, Shiping Experimental Equipment Co. Ltd., Shanghai, China) at $100 \mathrm{rpm}$. At 30 min, $1 \mathrm{~h}, 2 \mathrm{~h}, 3 \mathrm{~h}, 6 \mathrm{~h}, 12 \mathrm{~h}, 18 \mathrm{~h}$ and $24 \mathrm{~h}$, the aliquots were taken for analysis, and the same volume of blank medium was added.

\section{Study Design and Animal Model}

40 healthy New Zealand white rabbits $(2.0-2.5 \mathrm{~kg})$ were purchased from the Experimental Animal Center of Tongji Medical College of Huazhong University of Science and Technology. All experimental protocols were performed in accordance with the National Institutes of Health Guidelines and approved by the Ethics Committee of Tongji Medical College at Huazhong University of Science and Technology. The rabbits were maintained with free access to food and water.

The VX2 liver tumor models were established as described in the previous literature. ${ }^{20}$ Briefly, the VX2 tumor tissue was cut and separated into cubes at the size of $1 \mathrm{~mm}^{3}$ under sterile conditions, and then these pieces were stored in saline. Next, a tumor piece was embedded into each rabbit's hepatic left lateral lobe after anesthetized and then blocked with gelatin sponge to prevent the tumor mass from falling out or liver bleeding. Finally, the wound was sutured after ensuring no bleeding or complications. All rabbits were given intramuscular injection of penicillin for 3 days after the implantation of tumor piece. All animals were assigned randomly into four groups: NS group $(\mathrm{n}=10)$ was treated with $0.3 \mathrm{~mL}$ normal saline; $C B$ group $(n=10)$ was treated with $0.3 \mathrm{~mL}$ blank microsphere; ACB group $(n=10)$ was treated with $0.3 \mathrm{~mL}$ callispheres beads loaded with adriamycin (Hanhui Pharmaceutical Co. Ltd., Shanghai, China); DCB group $(n=10)$ was treated with $0.3 \mathrm{~mL}$ DCBs.

\section{MRI Scan Acquisition}

The liver tumor size was evaluated by magnetic resonance (MR, 3T, PHILIPS, Holland) on 14 days after modeling and 7 days after TACE. The scanning parameters used for conventional MR (T1WI and T2WI) imaging were TR 600/TE $8.0 \mathrm{~ms}$ and spin-echo in T1WI, TR 2725/TE 65 $\mathrm{ms}$, and fast spin-echo in T2WI, $12 \mathrm{~cm} \times 12 \mathrm{~cm}$ FOV, $3 \mathrm{~mm}$ thickness layer, and $152 \times 152$ matrix. $^{22}$ The long diameter (a) and short diameter (b) of the tumors were measured independently by two experienced radiologists. The tumor volume was calculated as $\mathrm{V}=\mathrm{a} \times \mathrm{b}^{2} / 2$, and the tumor growth rate was calculated as $\mathrm{V}_{1} / \mathrm{V}_{0} \times 100 \%\left(\mathrm{~V}_{1}\right.$ presented the tumor volume at $1 \mathrm{w}$ after TACE).

\section{Transarterial Chemoembolization Procedure}

After baseline MRI, the embolization procedures were performed immediately on digital subtraction arteriography (DSA, Siemens Medical Solutions, Munich, Germany). Under general anesthesia, the right femoral artery was exposed surgically, and a 4-F vascular sheath (Radiofocus Introducer II, Terumo Medical) was introduced in the exposed artery. After that, a 4-F Cobra catheter (Cook, Inc., Bloomington, IN) and a 2.4-F coaxial microcatheter system (Terumo, Tokyo, Japan) were used to select the celiac artery, hepatic artery and tumor-feeding artery successively for angiography. ${ }^{23}$ Then, the drugs or embolic materials were slowly injected by hand under careful real-time fluoroscopy to prevent nontarget embolization.

\section{Sample Collection}

The blood samples were collected through the ear artery of rabbits on $1 \mathrm{~d}, 3 \mathrm{~d}$ and $7 \mathrm{~d}$ after TACE. Serum was collected after centrifugation and stored at $-80{ }^{\circ} \mathrm{C}$. The bioanalysis machine (Lei Du Life Science Co. Ltd., Chemray 800, Shenzhen, China) was used to analyze the liver and kidney function, including alanine transaminase 
(ALT), aspartate transaminase (AST), creatinine ( $\mathrm{Cr}$ ) and blood urea nitrogen (BUN).

\section{Histopathologic Staining}

At 1 week after TACE, all rabbits were sacrificed immediately after MRI scanning, then the tumor tissues were removed and preserved in $4 \%$ formaldehyde for 24 hours before paraffin-embedded sections were made. For staining, the prepared paraffin sections were first deparaffinized and rehydrated. All samples were stained with hematoxylin and eosin $(\mathrm{HE})^{24}$ to assess tumor necrosis. The immunohistochemical (IHC) staining of proliferating cell nuclear antigen (PCNA) to display cell proliferation, HIF- $1 \alpha$ to compare the extent of tumor hypoxia, was performed. ${ }^{25}$ In addition, the immunofluorescence (IF) of CD31 and VEGF was performed to show the microvessel density (MVD) and VEGF expression. ${ }^{26}$

\section{Statistical Analysis}

All data were described as mean value \pm standard deviation and processed by SPSS statistics version 24.0 (SPSS Inc., Chicago, USA) and GraphPad Prism 8.0 (La Jolla, USA) software. The statistical methods of one-way ANOVA and LSD test were used to analyze the groups. A $P$ value $<0.05$ indicated significant difference. The histopathologic staining results were analyzed by Image J software 1.8.0 (Media Cybernetics, Rockville, MD, USA). The animal sample size was calculated by PASS 15.0 based on the previous study. ${ }^{27}$

\section{Results}

\section{DCBs Characterizations}

Donafenib loading efficiency peaked between 20 and 30 minutes, and the loading curve remained stable after 30 minutes (Figure 1B). The mean maximum loadability was $64.35 \%$. The donafenib release percentage gradually increased within $12 \mathrm{~h}$ and then kept stable (Figure 1C). The total drug release percentage reached $49.08 \%$ within $24 \mathrm{~h}$.

\section{Tumor Growth and Necrosis Rates}

The masses were clearly visible on MRI (Figure 2A and B), with hypointensity on T1WI and hyperintensity on T2WI, and the TACE process of VX2 liver tumor was shown in Figure $2 \mathrm{C}$ and D. Compared to the tumor growth rate of the NS group (253.49 $\pm 67.11 \%$ ), the tumor growth rates of the $\mathrm{CB}, \mathrm{ACB}$ and $\mathrm{DCB}$ groups were $192.17 \pm 63.01 \%$ $(\mathrm{P}=0.113), 193.27 \pm 47.25 \%(\mathrm{P}=0.110)$ and $105.80 \pm 36.42 \%$
$(\mathrm{P}=0.001)$, respectively (Figure $2 \mathrm{E}$ ). The tumor growth rate of the $\mathrm{DCB}$ group was lower than that in the $\mathrm{CB}(\mathrm{P}=0.031)$ and $\mathrm{ACB}(\mathrm{P}=0.022)$ groups, while there was no statistical significance between the $\mathrm{CB}$ and $\mathrm{ACB}$ groups $(\mathrm{P}=0.976)$ (Figure 2E). The tumor necrosis rate was defined as: $\frac{n T}{n T+T} *$ $100 \%$ (Figure 3A-D). Compared with the tumor necrosis rate of the NS group $(35.93 \pm 12.71 \%)$, those in the embolism groups were $75.59 \pm 23.05 \%(\mathrm{CB}, \mathrm{P}=0.004), 91.87$ $\pm 4.92 \% \quad(\mathrm{ACB}, \quad \mathrm{P}<0.001$ ), and $96.10 \pm 3.15 \%$ (DCB, $\mathrm{P}<0.001$ ), respectively (Figure $2 \mathrm{~F}$ ). Interestingly, there was no statistical difference between the $\mathrm{CB}, \mathrm{ACB}$ and $\mathrm{DCB}$ groups in the tumor necrosis rate (Figure $2 \mathrm{~F}$ ), implying that the beads, rather than the medicines, were the primary cause of tumor necrosis.

\section{IHC and IF Staining Results}

The quantitative analysis of IHC and IF staining revealed that, compared to the NS group, the expression of the HIF-1 $\alpha$ in all the rest of the embolism groups significantly enhanced (Figure 4A and B), which suggested these groups suffered a worsened hypoxic tumor environment after embolization. Although there was no difference in the expression of HIF-1 $\alpha$ between the $\mathrm{CB}, \mathrm{ACB}$ and $\mathrm{DCB}$ groups (Figure 4B), the VEGF expression of the DCB group was significantly lower than that of the $\mathrm{CB}$ and $\mathrm{ACB}$ group (Figure $5 \mathrm{~B}$ and $\mathrm{D}$ ). In addition, MVD in the DCB group was lower than that in the $\mathrm{CB}$ and $\mathrm{ACB}$ group (Figure 5A and $\mathrm{C}$ ), as well as the expression of PCNA (Figure 4A and $\mathrm{C}$ ). These shreds of evidence showed that the DCBs could inhibit the expression of VEGF, the formation of the new blood vessel and the tumor cell proliferation.

\section{Changes in Liver and Kidney Function}

As shown in Figure 6 and Table 1, except for the NS group, all the rest of the groups suffered a process of liver function damage and gradual recovery (Figure 6A and $\mathrm{B}$ ), while the kidney function was not affected (Figure 6C and D). The ACB group appeared to have more severe liver damage than the other groups. The ALT level of ACB was higher than that in the CB group (334.42 \pm 120.68 vs $161.19 \pm 33.91, \mathrm{P}=0.011)$ and $\mathrm{DCB}$ groups $(334.42 \pm 120.68$ vs $163.30 \pm 29.52, \mathrm{P}=0.012)$ on 7 days after TACE (Figure 6A, Table 1). The AST level of the ACB group was higher than that in the $\mathrm{CB}$ group on 3 days (194.76 \pm 104.18 vs $77.67 \pm 20.69, \mathrm{P}=0.032)$ and 7 days (146.17 \pm 71.74 vs $43.58 \pm 23.70, \mathrm{P}=0.032$ ) (Figure $6 \mathrm{~B}$, Table 1) after TACE. Furthermore, whereas the DCB group's AST level was greater one day after TACE than 

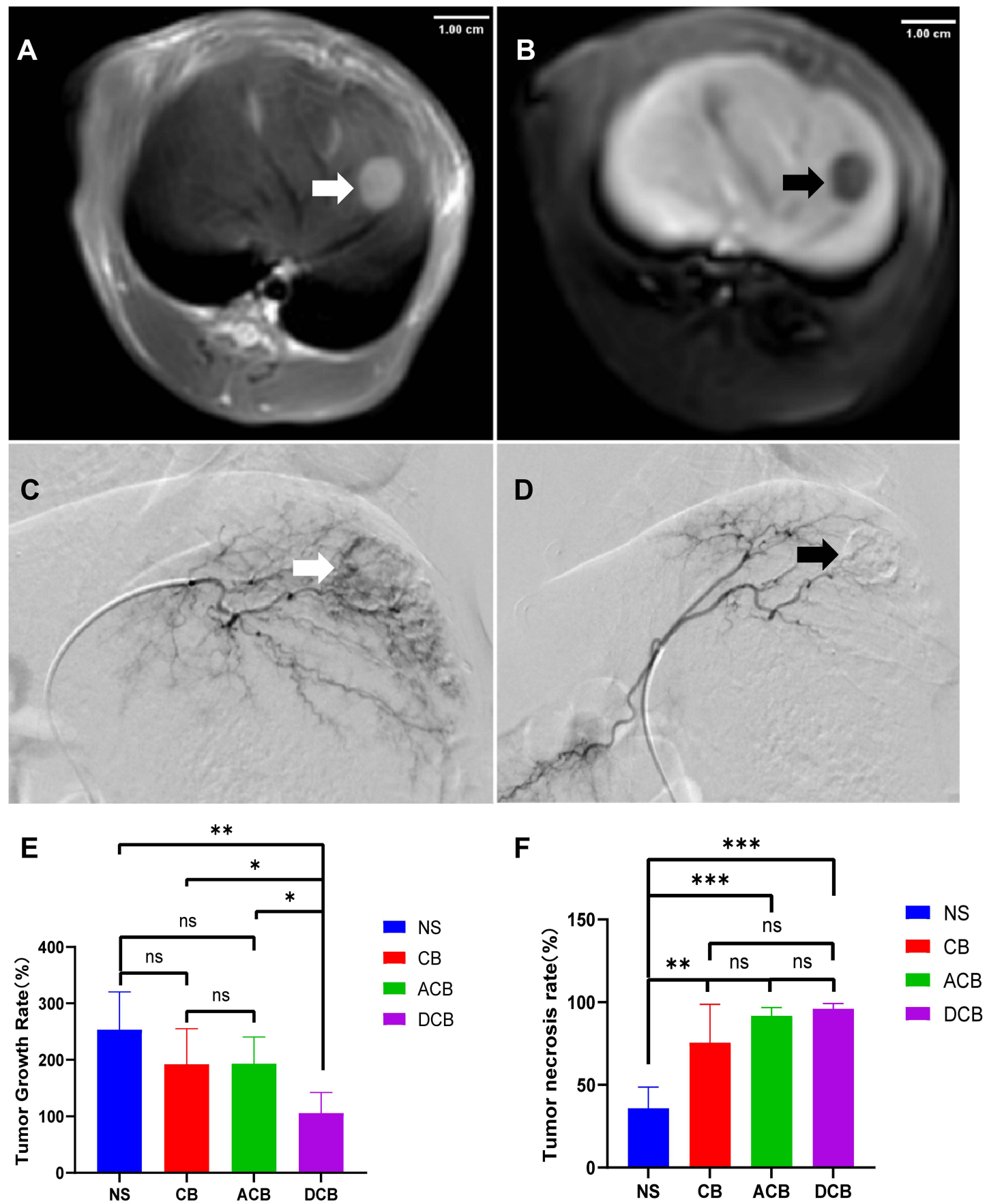

Figure 2 (A) Axial T2WI showing the mass with hyperintensity (white arrow); (B) axial TIWI showing the mass with hypointense (black arrow); (C) arterial angiography of a VX2 liver tumor before embolization; (D) after embolization with DCBs, the tumor staining disappeared; (E) the tumor growth rates of different groups after TACE; (F) The tumor necrosis rates of different groups after TACE. $* \mathrm{P}<0.05 ; * * \mathrm{P}<0.0 \mathrm{I} ; * * * \mathrm{P}<0.00 \mathrm{I}$.

the CB group (937.26 \pm 61.02 vs $548.42 \pm 232.71, \mathrm{P}=0.045)$, there was no statistical difference at 7 days $(\mathrm{P}=0.072)$ (Figure 6B, Table 1). These pieces of evidence indicated that the damage to liver function in the DCB group was similar to the $\mathrm{CB}$ group and somewhat better than the ACB group, and donafenib did not aggravate the liver damage.

\section{Discussion}

The basic principle of TACE was composed of the local ischemic necrosis caused by embolic material and the anticancer effects produced by chemotherapy. ${ }^{28}$ Increased tumor hypoxia after TACE, on the other hand, promotes both tumor resistance and the risk of tumor angiogenesis. ${ }^{7,29,30}$ Angiogenesis is necessary for tumor 


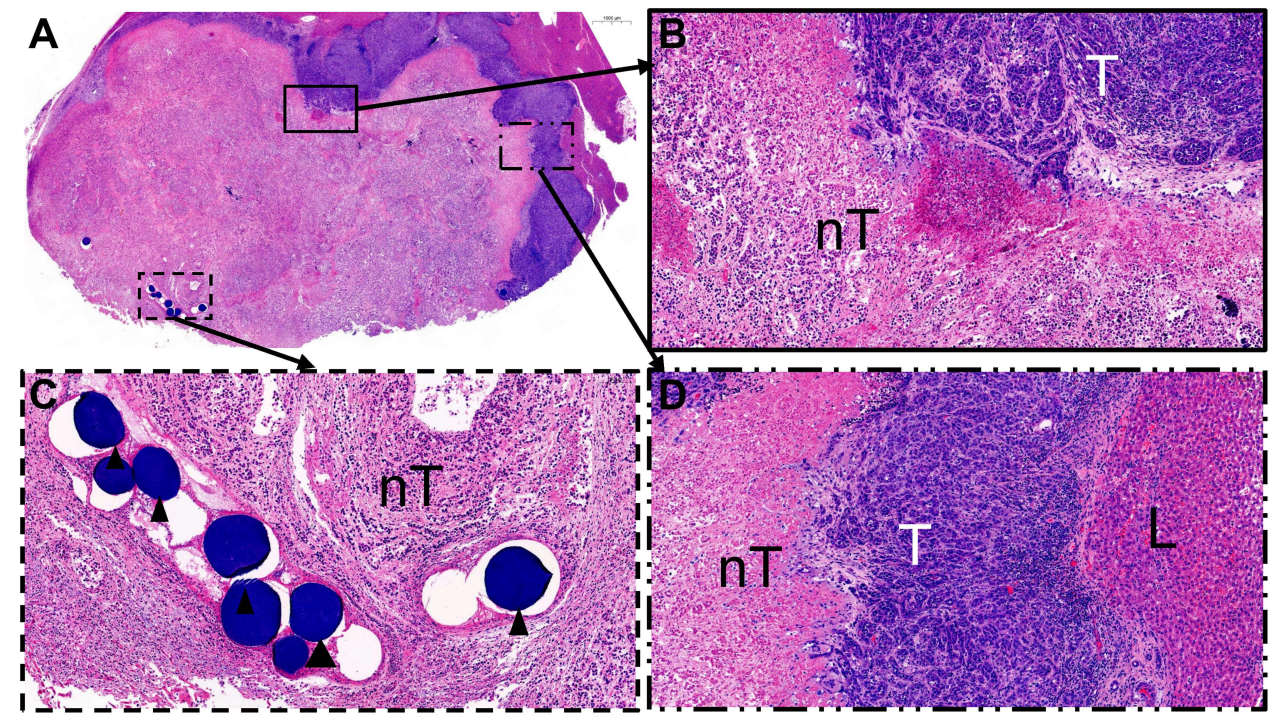

Figure 3 (A) Representative HE staining of the tumor after TACE. (B) Showing the necrosis of tumor tissue and liver tissue (I00x); (C) showing the callispheres beads (100x, black triangle); (D) showing three primary tissues (I00x).

Abbreviations: T, alive tumor tissue; $\mathrm{nT}$, necrosis tumor; L, liver tissue.

A

NS

CB

ACB

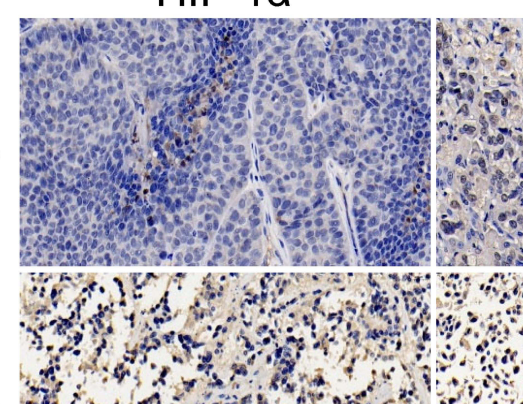

PCNA

भ.,
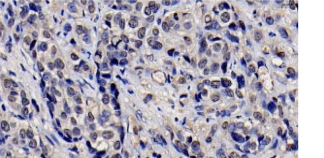

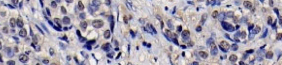

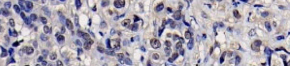
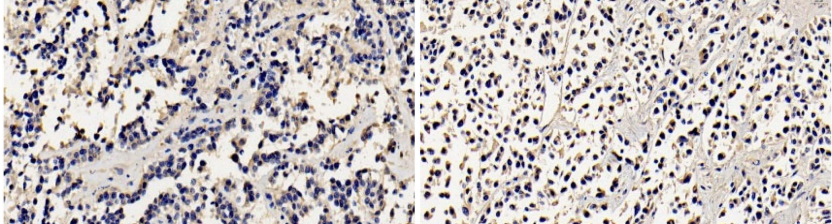

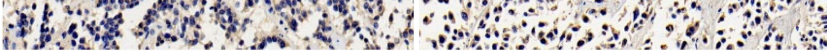

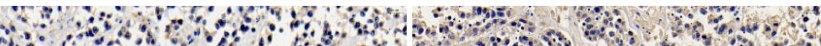

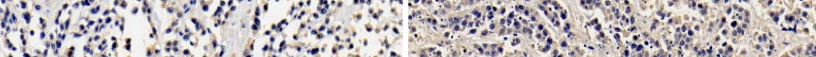

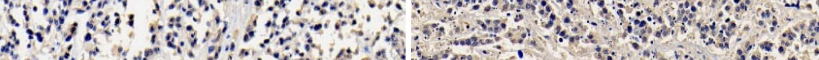
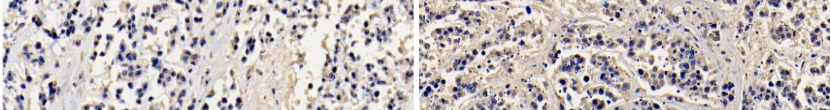
3.

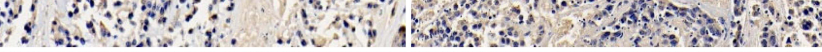

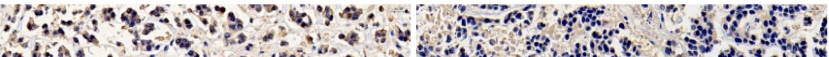

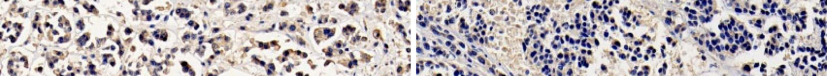

DCB

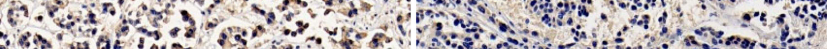
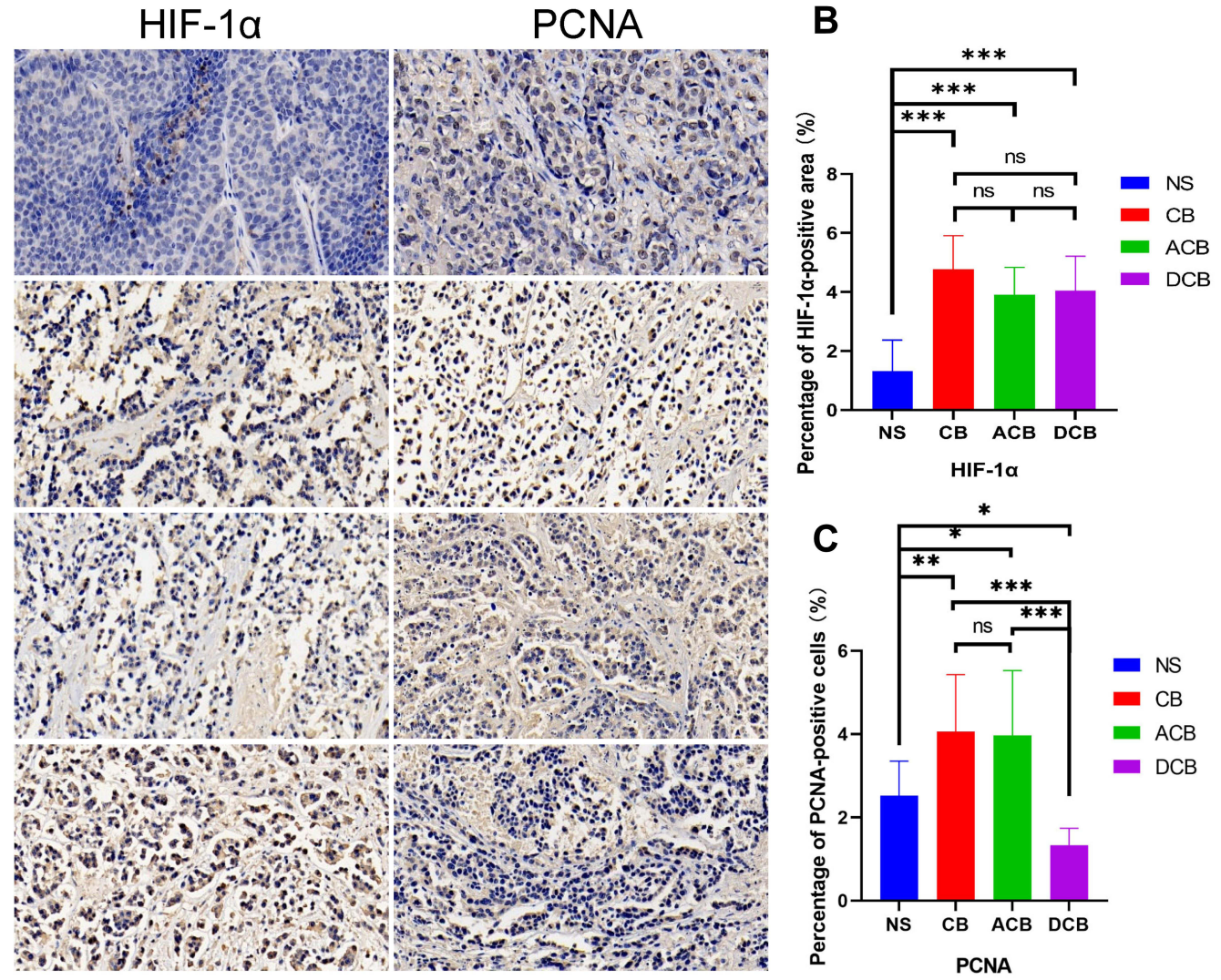

Figure 4 (A) Representative pictures of tumor HIF-I $\alpha$ and PCNA immunohistochemistry (400x); (B) quantitative analysis of HIF-I $\alpha$ positive results; (C) quantitative analysis of PCNA positive results. ${ }^{*} \mathrm{P}<0.05$; ${ }^{* *} \mathrm{p}<0.0 \mathrm{I}$; ${ }^{*} * * \mathrm{P}<0.00 \mathrm{I}$

invasion and migration and is an essential characteristic of tumor development, especially for HCC, a blood-rich tumor. ${ }^{27}$ In addition, the abnormal structure of tumor neovascularization leads to the development of edema, interstitial hypertension and further aggravates hypoxia, forming a vicious cycle of the hypoxic tumor 


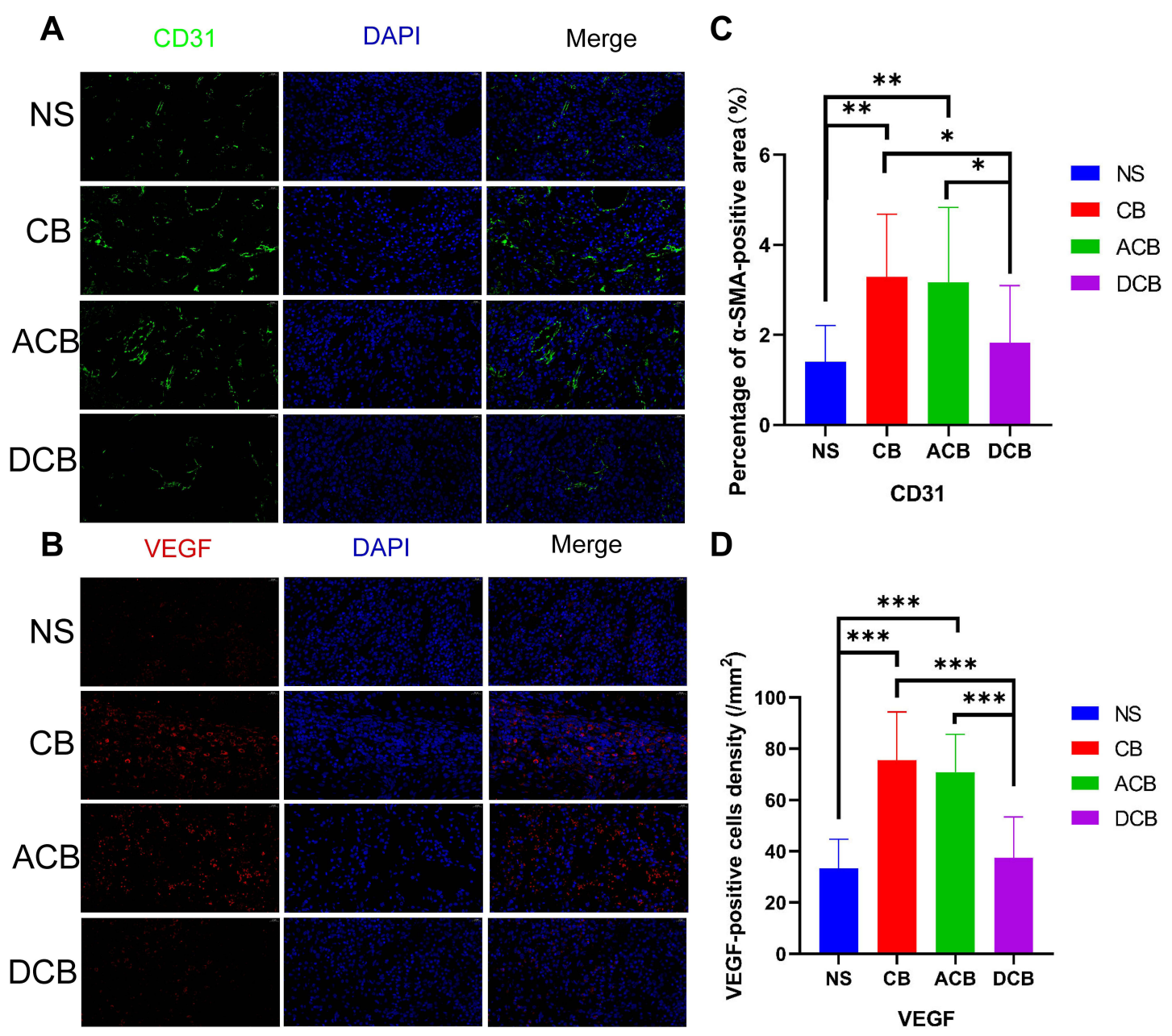

Figure 5 (A and B) Representative pictures of tumor CD3I (green) and VEGF (red) immunofluorescence (400x); (C and D) quantitative analysis of the expression of CD3। and VEGF. $* \mathrm{P}<0.05 ; * * \mathrm{p}<0.01 ; * * * \mathrm{P}<0.001$.

environment. ${ }^{31-33}$ Therefore, breaking this vicious circle after TACE is the key to inhibiting tumor blood vessel growth, thereby improving tumor recurrence or progression of HCC.

In this current study, we established a sustained-release system containing donafenib and explored the effects of DCBs on improving tumor angiogenesis. We discovered that DCBs can decrease the high expression of VEGF following embolization, which is important for tumor angiogenesis and tumor immunity escape. ${ }^{34,35}$ The data revealed that the DCB group had a higher rate of tumor necrosis, but tumor growth rate and MVD were lower, implying that donafenib prevented tumor growth via reducing tumor angiogenesis. We also noticed a decrease in tumor cell proliferation in the DCB group, which might be due to a decrease in VEGF or a direct pharmacological effect, but was ultimately linked to the effect of donafenib. The dose of donafenib applied in this study was about
$2 \mathrm{mg}$ per rabbit, a relatively small dose determined based on clinical trials and previous work ${ }^{16,20}$ and a suitable dose to improve the tumor microenvironment while avoiding liver damage. ${ }^{36,37}$

Administration of DCBs through the hepatic artery has unique advantages. Several studies have proved that this method can increase the drug concentration of the tumor foci while reducing the concentration of the drug in the serum, potentially decreasing the occurrence of side effects. ${ }^{15,24,38}$ Despite the lack of clinical trials, replacing TACE-loaded chemotherapeutics with antivascular drugs (eg, sorafenib, apatinib, sunitinib) has become a hot research direction in terms of basic research. ${ }^{19,20,38-40}$ Lipiodol and drug-eluting beads (DEBs) are the most commonly used drug delivery methods in these studies. Compared with lipiodol, the drugs can remain in the tumor foci longer due to the sustained release profiles of DEBs. ${ }^{41,42}$ Furthermore, lipiodol loading targeted drugs 
A

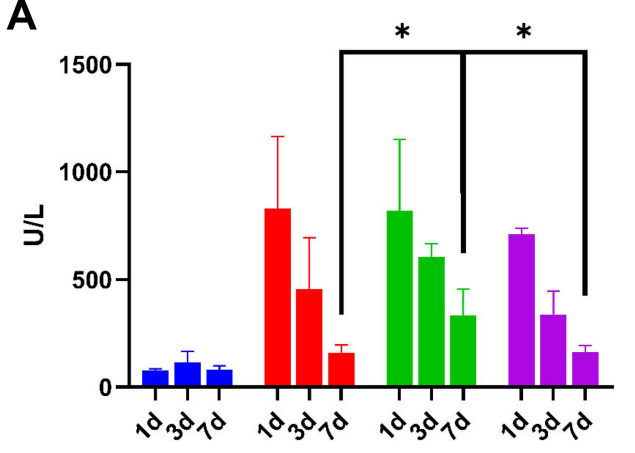

C

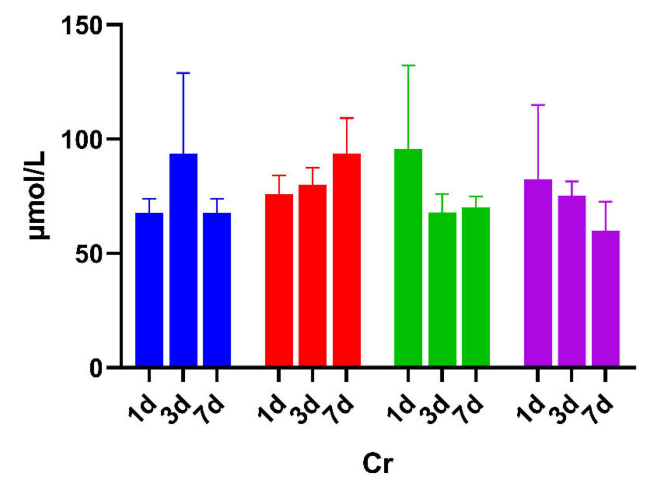

B
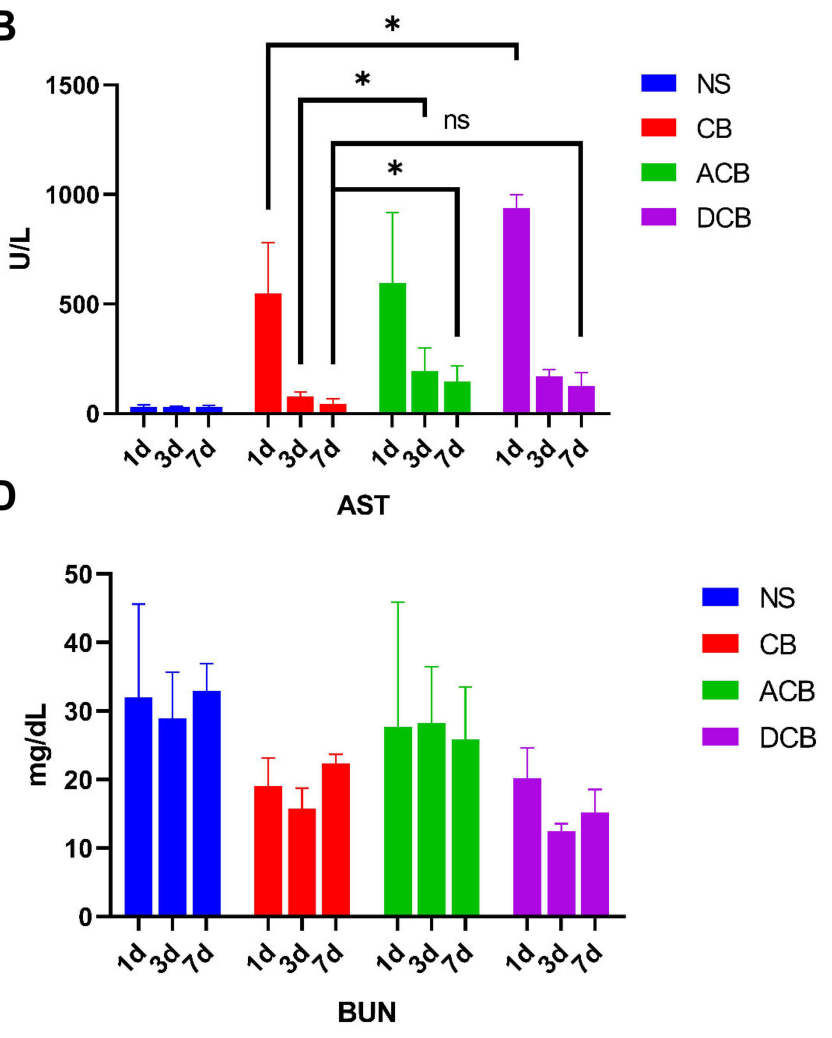

Figure 6 Biochemical tests of VX2 liver tumor-bearing rabbits after TACE; (A) ALT levels; (B) AST levels; (C) Cr values; (D) BUN values. *P<0.05.

damaged liver function more severely than lipiodol alone, ${ }^{23,38,39}$ while targeted drugs loaded with DEBs will not aggravate liver damage, ${ }^{19,28}$ which agrees with our experimental results. This finding could be explained by the fact that DEBs are solid embolic materials, ${ }^{43,44}$ resulting in less ectopic embolism.
It was worth mentioning that adriamycin appeared to do nothing but worsen liver function, considering there was no statistical difference between the $\mathrm{CB}$ group and the ACB group on both the tumor growth and necrosis rate. Several studies have found that chemoembolization was not better than bland embolization, which suggests that the role of

Table I Temporal Evolution of Liver and Kidney Function Parameters

\begin{tabular}{|l|c|c|c|c|c|}
\hline Group & Time & ALT (U/L) & AST (U/L) & Cr ( $\mathbf{m m o l} / \mathbf{L})$ & BUN (mg/dL) \\
\hline NS & Id & $78.4 \pm 6.8$ & $30.0 \pm 9.9$ & $67.7 \pm 6.3$ & $32.0 \pm 13.6$ \\
& 3d & $113.8 \pm 53.4$ & $29.7 \pm 5.1$ & $93.5 \pm 35.4$ & $29.0 \pm 6.8$ \\
& 7d & $81.4 \pm 18.4$ & $30.1 \pm 8.1$ & $67.8 \pm 6.1$ & $32.9 \pm 4.0$ \\
\hline CB & Id & $829.9 \pm 335.7$ & $548.4 \pm 232.7$ & $76.1 \pm 8.1$ & $19.1 \pm 4.1$ \\
& 3d & $456.6 \pm 239.3$ & $77.7 \pm 20.7$ & $80.1 \pm 7.4$ & $15.8 \pm 3.0$ \\
& 7d & $161.2 \pm 33.9$ & $43.6 \pm 23.7$ & $93.9 \pm 15.4$ & $22.4 \pm 1.3$ \\
\hline ACB & Id & $880.7 \pm 227.2$ & $596.9 \pm 320.3$ & $95.8 \pm 36.6$ & $27.7 \pm 18.2$ \\
& 3d & $545.5 \pm 110.2$ & $194.8 \pm 104.2$ & $67.9 \pm 8.2$ & $28.3 \pm 8.1$ \\
& 7d & $334.4 \pm 120.7$ & $146.2 \pm 71.7$ & $70.2 \pm 4.8$ & $25.9 \pm 7.6$ \\
\hline DCB & Id & $712.2 \pm 26.2$ & $937.3 \pm 61.0$ & $82.4 \pm 32.6$ & $20.2 \pm 4.4$ \\
& 3d & $337.8 \pm 109.6$ & $169.1 \pm 31.5$ & $75.3 \pm 6.2$ & $12.5 \pm 1.0$ \\
& 7d & $163.3 \pm 29.5$ & $125.8 \pm 60.5$ & $60.1 \pm 12.6$ & $15.2 \pm 3.4$ \\
\hline
\end{tabular}

Abbreviations: AST, alanine transaminase; AST, aspartate transaminase; $\mathrm{Cr}$, creatinine; BUN, urea nitrogen. 
chemotherapy drugs used in TACE is currently unclear and controversial. $^{45-47}$ A previous animal experiment has shown that doxorubicin was linked with more tumor necrosis, ${ }^{48}$ which contradicts the findings of this investigation. The possible reason is that the effect of microspheres has a greater effect on tumor necrosis in this experiment (Figure 2F), and adriamycin has no effect on necrotic tumor cells.

There are also conflicts between the results of fundamental experiments and clinical research. Although the chemotherapeutic drugs used in TACE have a definite toxic effect on HCC cells, ${ }^{49}$ the clinical benefit is not very clear, ${ }^{45}$ which could be due to chemotherapeutic drug penetration into the tumor being insufficient. ${ }^{50}$ In this study, we compared the effectiveness and safety of DCBs and ACBs (conventional TACE) embolization in a preclinical cancer model and proved that replacing the chemotherapeutic drugs with antivascular drugs can achieve better treatment effect. ${ }^{19,39}$ More importantly, we observed a decrease in the expression of VEGF and MVD in the DCB group, both of which are related to the prognosis of patients. ${ }^{51,52}$

There were several limitations in the study: firstly, the number of animals in each group was limited. Secondly, the rabbit VX2 liver tumor is a perfect model for transhepatic artery embolization but an imperfect surrogate for human HCC because it is an adenocarcinoma cell line rather human HCC cell line. This study set out to load donafenib onto callispheres microspheres and we found that donafenib can be released slowly in this system. When the DCBs was applied to the pre-clinical VX2 liver tumor model via the hepatic artery, donafenib can alleviate tumor angiogenesis and reduce tumor cell proliferation by reducing the expression of VEGF but did not aggravate the damage to liver function.

To summarize, DCBs embolization via hepatic artery is an effective and safe treatment that improves the tumor angiogenesis after embolization of HCC.

\section{Acknowledgments}

This work was financially supported by grants from the National Natural Science Foundation of China (No. 81873917 and 81471766). We are grateful for the help from Zelgen Medicine Co. Ltd., Jiangsu, China.

\section{Disclosure}

The authors report no conflicts of interest in this work.

\section{References}

1. Gnutzmann D, Kortes N, Sumkauskaite M, et al. Transvascular therapy of Hepatocellular Carcinoma (HCC), status and developments. Minim Invasive Ther Allied Technol. 2018;27 (2):69-80. doi:10.1080/13645706.2018.1432489

2. Chang Y, Jeong SW, Young Jang J, et al. Recent updates of transarterial chemoembolilzation in hepatocellular carcinoma. Int J Mol Sci. 2020;21(21):8165. doi:10.3390/ijms21218165

3. Liu K, Min X-L, Peng J, et al. The changes of HIF-1 $\alpha$ and VEGF expression after TACE in patients with hepatocellular carcinoma. J Clin Med Res. 2016;8(4):297-302. doi:10.14740/jocmr2496w

4. Palazon A, Tyrakis PA, Macias D, et al. An HIF-1 $\alpha /$ VEGF-a axis in cytotoxic T cells regulates tumor progression. Cancer Cell. 2017;32 (5):669-683. doi:10.1016/j.ccell.2017.10.003

5. Petrillo M, Patella F, Pesapane F, et al. Hypoxia and tumor angiogenesis in the era of hepatocellular carcinoma transarterial loco-regional treatments. Future Oncol. 2018;14(1):2957-2967. doi:10.2217/fon2017-0739

6. Sergio A, Cristofori C, Cardin R, et al. Transcatheter arterial chemoembolization (TACE) in hepatocellular carcinoma (HCC): the role of angiogenesis and invasiveness. Am J Gastroenterol. 2008;103 (4):914-921. doi:10.1111/j.1572-0241.2007.01712.x

7. Tezuka M, Hayashi K, Kubota K, et al. Growth rate of locally recurrent hepatocellular carcinoma after transcatheter arterial chemoembolization: comparing the growth rate of locally recurrent tumor with that of primary hepatocellular carcinoma. Dig Dis Sci. 2007;52(3):783-788. doi:10.1007/s10620-006-9537-y

8. Britten CD, Gomes AS, Wainberg ZA, et al. Transarterial chemoembolization plus or minus intravenous bevacizumab in the treatment of hepatocellular cancer: a pilot study. BMC Cancer. 2012;12(1):16. doi:10.1186/1471-2407-12-16

9. Fang P, Hu J-H, Cheng Z-G, et al. Efficacy and safety of bevacizumab for the treatment of advanced hepatocellular carcinoma: a systematic review of phase II trials. PLoS One. 2012;7(12): e49717. doi:10.1371/journal.pone.0049717

10. Sakr OS, Berndt S, Carpentier G, et al. Arming embolic beads with anti-VEGF antibodies and controlling their release using LbL technology. J Control Release. 2016;224(1873):199-207. doi:10.1016/j.jconrel.2016.01.010

11. Kudo MA, Ueshima K, Ikeda M, et al. Randomised, multicentre prospective trial of transarterial chemoembolisation (TACE) plus sorafenib as compared with TACE alone in patients with hepatocellular carcinoma: TACTICS trial. Gut. 2020;69(8):1492-1501. doi:10.1136/gutjnl-2019-318934

12. Kudo M, Imanaka K, Chida N, et al. Phase III study of sorafenib after transarterial chemoembolisation in Japanese and Korean patients with unresectable hepatocellular carcinoma. Eur J Cancer. 2011;47 (14):2117-2127. doi:10.1016/j.ejca.2011.05.007

13. Lencioni R, Llovet JM, Han G, et al. Sorafenib or placebo plus TACE with doxorubicin-eluting beads for intermediate stage HCC: the SPACE trial. $J$ Hepatol. 2016;64(5):1090-1098. doi:10.1016/j. jhep.2016.01.012

14. Li N, Chen B, Lin R, et al. The earlier, the better: the effects of different administration timepoints of sorafenib in suppressing the carcinogenesis of VEGF in rats. Cancer Chemother Pharmacol. 2018;81(1):207-216. doi:10.1007/s00280-017-3493-4

15. Ai L, Xu Z, Yang B, et al. Sorafenib-associated hand-foot skin reaction: practical advice on diagnosis, mechanism, prevention, and management. Expert Rev Clin Pharmacol. 2019;12(12):1121-1127. doi:10.1080/17512433.2019.1689122

16. Qin SA, Bi F, Gu S, et al. Donafenib versus sorafenib in first-line treatment of unresectable or metastatic hepatocellular carcinoma: a randomized, open-label, parallel-controlled phase II-III trial. J Clin Oncol. 2021;39(27):JCO2100163. 
17. Li X, Qiu M, Wang S, et al. A phase I dose-escalation, pharmacokinetics and food-effect study of oral donafenib in patients with advanced solid tumours. Cancer Chemother Pharmacol. 2020;85 (3):593-604. doi:10.1007/s00280-020-04031-1

18. Liu J, Li X, Zhang H. Safety, pharmacokinetics and efficacy of donafenib in treating advanced hepatocellular carcinoma: report from a phase 1b trial. Die Pharmazie. 2019;74(11):688-693. doi:10.1691/ph.2019.9626

19. Fuchs K, Bize PE, Dormond O, et al. Drug-eluting beads loaded with antiangiogenic agents for chemoembolization: in vitro sunitinib loading and release and in vivo pharmacokinetics in an animal model. J Vasc Interv Radiol. 2014;25(3):379. doi:10.1016/j.jvir.2013.11.039

20. Shi Q, Lu Y, Huang S, et al. Apatinib-loaded CalliSpheres Beads for embolization in a rabbit VX2 liver tumor: characterization in vitro, pharmacokinetics and tumor response in vivo. Drug Deliv. 2020;27 (1):1301-1307. doi:10.1080/10717544.2020.1818881

21. Chen Q, Sun Y, Dai H, Guo P, Hou S, Sha X Assessment of irinotecan loading and releasing profiles of a novel drug-eluting microsphere (CalliSpheres) in vitro. Cancer Biother Radiopharm. 2020;3805. doi:10.1089/cbr.2020.3805

22. Qian T, Chen M, Gao F, et al. Diffusion-weighted magnetic resonance imaging to evaluate microvascular density after transarterial embolization ablation in a rabbit VX2 liver tumor model. Magn Reson Imaging. 2014;32(8):1052-1057. doi:10.1016/j.mri.2014.05.011

23. Seidensticker M, Streit S, Nass N, et al. Modified transarterial chemoembolization with locoregional administration of sorafenib for treating hepatocellular carcinoma: feasibility, efficacy, and safety in the VX-2 rabbit liver tumor model. Diagn Interv Radiol. 2016;22 (4):378-384. doi:10.5152/dir.2016.15462

24. Hong K, Khwaja A, Liapi E, et al. New intra-arterial drug delivery system for the treatment of liver cancer: preclinical assessment in a rabbit model of liver cancer. Clin Cancer Res. 2006;12 (8):2563-2567. doi:10.1158/1078-0432.CCR-05-2225

25. Borde T, Laage Gaupp F, Geschwind J-F, et al. Idarubicin-loaded ONCOZENE drug-eluting bead chemoembolization in a rabbit liver tumor model: investigating safety, therapeutic efficacy, and effects on tumor microenvironment. J Vasc Interv Radiol. 2020;31(10):17061716 e1. doi:10.1016/j.jvir.2020.04.010

26. Zhou H, Li X, Yin Y, et al. The proangiogenic effects of extracellular vesicles secreted by dental pulp stem cells derived from periodontally compromised teeth. Stem Cell Res Ther. 2020;11(1):110. doi:10.1186/ s13287-020-01614-w

27. Zhou C, Shi Q, Liu J, et al. Effect of inhibiting tumor angiogenesis after embolization in the treatment of HCC with apatinib-loaded $p$ (N-Isopropyl-Acrylamide-co-Butyl methyl acrylate) temperaturesensitive nanogel. J Hepatocell Carcinoma. 2020;7(2253):447-456. doi:10.2147/JHC.S282209

28. Li X, Yu H, Huang Y, et al. Preparation of microspheres encapsulating sorafenib and catalase and their application in rabbit VX2 liver tumor. Biomed Pharmacother. 2020;129(1950):110512. doi:10.1016/ j.biopha.2020.110512

29. Pleguezuelo M, Marelli L, Misseri M, et al. TACE versus TAE as therapy for hepatocellular carcinoma. Expert Rev Anticancer Ther. 2008;8(10):1623-1641. doi:10.1586/14737140.8.10.1623

30. Kim YB, Park C, Fau park YN, Park C. Increased proliferation activities of vascular endothelial cells and tumour cells in residual hepatocellular carcinoma following transcatheter arterial embolization. Histopathology. 2001;38(2):160-166. doi:10.1046/ j.1365-2559.2001.01064.x

31. Liu K, Zhang X, Xu W, et al. Targeting the vasculature in hepatocellular carcinoma treatment: starving versus normalizing blood supply. Clin Transl Gastroenterol. 2017;8(6):e98. doi:10.1038/ ctg. 2017.28

32. Simon T, Gagliano T, Giamas G. Direct effects of anti-angiogenic therapies on tumor cells: VEGF signaling. Trends Mol Med. 2017;23 (3):282-292. doi:10.1016/j.molmed.2017.01.002
33. Vasudev NS, Reynolds AR. Anti-angiogenic therapy for cancer: current progress, unresolved questions and future directions. Angiogenesis. 2014;17(3):471-494. doi:10.1007/s10456-014-9420-y

34. Apte RS, Chen DS, Ferrara N. VEGF in signaling and disease: beyond discovery and development. Cell. 2019;176(6):1248-1264. doi:10.1016/j.cell.2019.01.021

35. Morse MA, Sun W, Kim R, et al. The role of angiogenesis in hepatocellular carcinoma. Clin Cancer Res. 2019;25(3):912-920. doi:10.1158/1078-0432.CCR-18-1254

36. Chatziioannou AN, Siskos AP, Loxas D, et al. Transarterial embolization with sorafenib in animal livers: a pharmacokinetics study. J Vasc Interv Radiol. 2013;24(11):1657-63 e1. doi:10.1016/j. jvir.2013.08.007

37. Zhao S, Ren S, Jiang T, et al. Low-dose apatinib optimizes tumor microenvironment and potentiates antitumor effect of PD-1/PD-L1 blockade in lung cancer. Cancer Immunol Res. 2019;7(4):630-643. doi:10.1158/2326-6066.CIR-17-0640

38. Parvinian A, Casadaban LC, Hauck ZZ, et al. Pharmacokinetic study of conventional sorafenib chemoembolization in a rabbit VX2 liver tumor model. Diagn Interv Radiol. 2015;21(3):235-240. doi:10.5152/ dir.2014.14394

39. Kim GM, Kim MD, Kim DY, et al. Transarterial chemoembolization using sorafenib in a rabbit VX2 liver tumor model: pharmacokinetics and antitumor effect. J Vasc Intervent Radiol. 2016;27(7):1086-1092. doi:10.1016/j.jvir.2016.02.032

40. Bize P, Duran R, Fuchs K, et al. Antitumoral effect of sunitinib-eluting beads in the rabbit VX2 tumor model. Radiology. 2016;280(2):425-435. doi:10.1148/radiol.2016150361

41. Zhang S, Huang C, Li Z, et al. Comparison of pharmacokinetics and drug release in tissues after transarterial chemoembolization with doxorubicin using diverse lipiodol emulsions and CalliSpheres Beads in rabbit livers. Drug Deliv. 2017;24(1):1011-1017. doi:10.1080/10717544.2017.1344336

42. Han S, Zhang X, Zou L, et al. Does drug-eluting bead transcatheter arterial chemoembolization improve the management of patients with hepatocellular carcinoma? A meta-analysis. PLoS One. 2014;9 (1932):e102686. doi:10.1371/journal.pone.0102686

43. Melchiorre F, Patella F, Pescatori L, et al. DEB-TACE: a standard review. Future Oncol. 2018;14(8):2969-2984. doi:10.2217/fon-2018-0136

44. Lammer J, Malagari K, Vogl T, et al. Prospective randomized study of doxorubicin-eluting-bead embolization in the treatment of hepatocellular carcinoma: results of the PRECISION V study. Cardiovasc Intervent Radiol. 2010;33(1):41-52. doi:10.1007/s00270-009-9711-7

45. Boulin M, Guiu B. Chemoembolization or bland embolization for hepatocellular carcinoma: the question is still unanswered. $J$ Clin Oncol. 2017;35(2):256-257. doi:10.1200/JCO.2016.67.2915

46. Roth GA, Benhamou M, Teyssier Y, et al. Comparison of trans-arterial chemoembolization and bland embolization for the treatment of hepatocellular carcinoma: a propensity score analysis. Cancers (Basel). 2021;13(4):812. doi:10.3390/cancers 13040812

47. Brown KT, Do RK, Gonen M, et al. Randomized trial of hepatic artery embolization for hepatocellular carcinoma using doxorubicin-eluting microspheres compared with embolization with microspheres alone. J Clin Oncol. 2016;34(17):2046-+. doi:10.1200/ JCO.2015.64.0821

48. Gaba RC, Emmadi R, Parvinian A, et al. Correlation of doxorubicin delivery and tumor necrosis after drug-eluting bead transarterial chemoembolization of rabbit VX2 liver tumors. Radiology. 2016;280(3):752-761. doi:10.1148/radiol.2016152099

49. Boulin M, Guiu S, Chauffert B, et al. Screening of anticancer drugs for chemoembolization of hepatocellular carcinoma. Anticancer Drugs. 2011;22(8):741-748. doi:10.1097/CAD.0b013e328346a0c5

50. Liang B, Xiong F, Wu H, et al. Effect of transcatheter intraarterial therapies on the distribution of Doxorubicin in liver cancer in a rabbit model. PLoS One. 2013;8(10):e76388. doi:10.1371/journal. pone. 0076388 
51. Cao G, Li X, Qin C, et al. Prognostic value of VEGF in hepatocellular carcinoma patients treated with sorafenib: a meta-analysis. Med Sci Monit. 2015;21(1643):3144-3151. doi:10.12659/MSM.894617
52. Chen ZY, Wei W, Guo ZX, et al. Morphologic classification of microvessels in hepatocellular carcinoma is associated with the prognosis after resection. J Gastroenterol Hepatol. 2011;26(5):866-874. doi:10.1111/j.1440-1746.2010.06511.x

\section{Publish your work in this journal}

The Journal of Hepatocellular Carcinoma is an international, peerreviewed, open access journal that offers a platform for the dissemination and study of clinical, translational and basic research findings in this rapidly developing field. Development in areas including, but not limited to, epidemiology, vaccination, hepatitis therapy, pathology and molecular tumor classification and prognostication are all considered for publication. The manuscript management system is completely online and includes a very quick and fair peer-review system, which is all easy to use. Visit http://www.dovepress.com/ testimonials.php to read real quotes from published authors.

Submit your manuscript here: https://www.dovepress.com/journal-of-hepatocellular-carcinoma-journal 